\title{
PAPR and BER Performances of OFDM System with Novel Tone Reservation Technique Over Frequency Non-Selective Fading Channel
}

\author{
Moftah Ali*, Raveendra K. Rao, Vijay Parsa \\ Innovation Centre for Information Engineering (ICIE), Faculty of Engineering, Department of Electrical and Computer Engineering,
} University of Western Ontario, London, Ontario, N6A 5B9, Canada

\begin{tabular}{l} 
A R T I C L E I N F O \\
\hline Article history: \\
Received: 21 December, 2018 \\
Accepted: 08 February, 2019 \\
Online : 20 February, 2019 \\
\hline Keywords: \\
Orthogonal Frequency Division \\
Multiplexing \\
Tone Reservation \\
Pseudo-random Sequence \\
\end{tabular}

\begin{abstract}
A B S T R A C T
An Orthogonal Frequency Division Multiplexing (OFDM) system with Quadrature Phase Shift Keying (QPSK) mapper is considered. A novel low-complexity Tone Reservation (TR) technique is proposed for reduction of Peak-to-Average Power Ratio (PAPR) of the system. The technique is easy-to-implement and minimizes the search space of phases of reserved tones in the system. The ability of PAPR reduction of this proposed TR technique is assessed and compared with conventional TR technique that uses Pseudo Noise (PN) sequences to determine phases of reserved tones. The simulation results illustrate that the proposed TR technique is nearly the same as that of the conventional TR technique in terms of PAPR reduction capability but with reduced complexity. The Bit Error Rate (BER) performance of QPSK-OFDM system with the novel TR scheme over frequency non-selective Rayleigh fading channel is also determined and illustrated.
\end{abstract}

\section{Introduction}

Multicarrier modulation such as OFDM is extensively adopted in numerous communication systems and standards. For example, Digital Audio Broadcasting (DAB) [1], IEEE set of standards that includes wireless local area network 802.11a [2], wireless broadband standard 802.16 [3], and mobile broadband wireless access 802.20 [4]. In addition, OFDM is the core technique for 3GPP [5] and Large Term Evolution (LTE) [6] etc. OFDM systems are attractive due to: i) their immunity to multipath fading and impulse noise; ii) elimination of complex equalizer; and iii) efficient implementation using IFFT/FFT for modulation/demodulation. OFDM system has one significant drawback that is the transmitted signals exhibit high values of PAPR. As a result, a High Power Amplifier (HPA) with sufficient linear amplification range is required in the system; otherwise, the transmitted signal would be nonlinearly distorted resulting in loss of subcarrier orthogonality, and hence BER degradation of the system. Thus, it is important to consider an appropriate PAPR reduction technique in an OFDM for it to be cost effective as well as energy efficient. In the literature, several methods have been proposed and investigated, which can mitigate PAPR problem in an OFDM system, such as Clipping and Filtering (CF) [7], weighting [8], Selective Mapping (SLM) [9], [10], Active
Constellation Extension (ACE) [11], Partial Transmit Sequence [12], [13], Tone Reservation (TR) [14], Tone Injection [14], coding [15] etc. Each technique used in an OFDM system offers its own computational complexity, PAPR reduction capability, amount to side information required to be exchanged between transmitter and receiver, and BER performance of the system [16], [17], [18].

The focus in this paper is on TR technique for reduction of PAPR in an OFDM system that requires: i) fewer computations compared to TR techniques available in the literature and ii) no side information to be exchanged between transmitter and receiver. This method was briefly presented in [19]. In this paper, this work is extended and examined in more detail and is compared with the well-known SLM technique for PAPR reduction [18]. It is noted that SLM is a distortion-less technique and requires side information (SI) to be conveyed to receiver. In addition, the QPSK-OFDM system with TR method introduced in the paper is examined for BER performance over Rayleigh fading channel.

In the paper, a novel TR technique with low-complexity is presented for reduction of PAPR in an OFDM system; the proposed scheme is easy-to-use and minimizes the search space of phase sequences. The technique achieves approximately same performance as that of TR technique using conventional PN

*Corresponding Author: Moftah Ali, Email: mali254@uwo.ca 
M. Ali et al. / Advances in Science, Technology and Engineering Systems Journal Vol. 4, No. 1, 225-230 (2019)

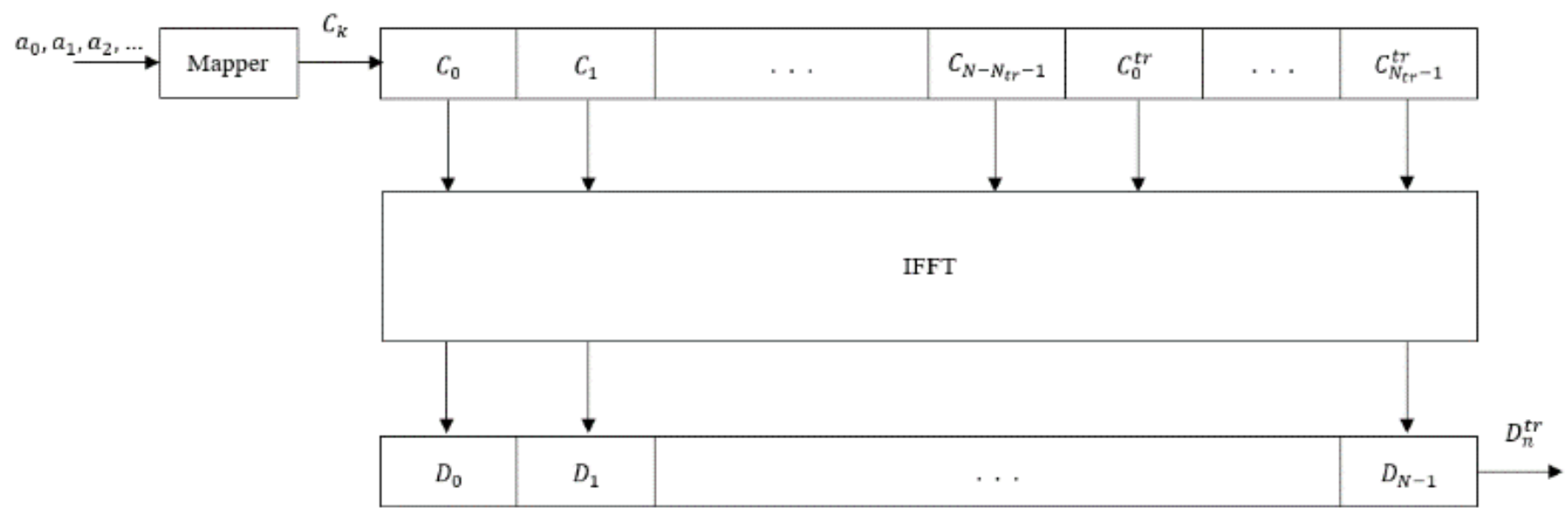

Figure 1: Block diagram for generation of OFDM signal with TR technique.

sequence. The simulation results are provided for a 128-subcarrier QPSK-OFDM system, as a function of numbers of tones reserved The probability of bit error performance of the system is also presented.

In section 2, OFDM signal and conventional TR technique are explained. Section 3 describes the TR method introduced in the paper. In section 4, OFDM receiver is discussed and in section 5, BER of the system in frequency-non-selective Rayleigh fading channel is presented. In section 6, numerical results are presented and discussed. The paper concluded in section 7 .

\section{OFDM Signal Model and TR Technique}

\subsection{OFDM Signal and its PAPR}

An OFDM signal is a summation of $N$ (number of subcarriers or tones) independently modulated complex sinusoidal signals, and is given by:

$$
D(t)=\frac{1}{N} \sum_{k=0}^{N-1} C_{k} e^{j 2 \pi f_{k} t}, \quad 0 \leq t \leq T_{s}
$$

where $j=\sqrt{-1}, T_{s}$ is the symbol duration and, $C_{k}, k=$ $0,1, \ldots, N-1$, are the complex data. When QPSK mapper is considered, $C_{k} \in\{ \pm 1 \pm j\}$ and the subcarrier frequencies are $f_{k}=k / T_{s}, k=0,1, \ldots, N-1$. When signal in (1) is sampled at Nyquist rate, discrete OFDM signal obtained and is given by:

$$
D_{n}=D\left(n 2 T_{b}\right)=\frac{1}{N} \sum_{k=0}^{N-1} C_{k} e^{\frac{j \pi n k}{N}}, n=0,1, \ldots N-1
$$

The PAPR of signal in (1) is:

$$
P A P R=\frac{\max }{\frac{0 \leq t \leq T_{s}}{T_{s}} \int_{0}^{T_{s}}|D(t)|^{2} d t}
$$

Equivalently, PAPR in (3) is given by:

$$
P A P R=\frac{\max \left\{\left|D_{n}\right|^{2}, n=0,1, \ldots, L N-1\right\}}{\frac{1}{N L} \sum_{n=0}^{N L-1}\left|D_{n}\right|^{2}}
$$

where $L(\geq 4)$ is the oversampling factor.

\subsection{TR Technique}

In TR method, $N_{t r}$ of $N$ tones, are reserved and used for PAPR reduction [20]. Figure 1 illustrates a block diagram for generation of OFDM signal with TR technique. The ratio $R=N_{t r} / N$ is typically kept small. Thus, the OFDM signal with tone reservation technique can be expressed as:

$$
\begin{gathered}
D_{n}^{T R}=D_{n}+D_{n}^{t r} \\
D_{n}^{T R}=\frac{1}{N} \sum_{k=0}^{N-1}\left(C_{k}+C_{k}^{t r}\right) e^{j 2 \pi k n / N}, \quad n=0,1, \ldots, N-1
\end{gathered}
$$

where $C_{k}=\left[C_{0}, C_{1}, \ldots, C_{N-1}\right]$ and $C^{t r}=\left[C_{0}^{t r}, C_{1}^{t r}, \ldots, C_{N-1}^{t r}\right]$ represent frequency domain vectors associated with data and reserved tones. The reserved tones' locations are denoted by the index $\mathcal{R}^{t r}=\left\{i_{0}, i_{1}, \ldots, \quad i_{N_{t r}-1}\right\}$ where $0 \leq i_{0} \leq i_{1} \leq \cdots \leq$ $i_{N_{t r}-1} \leq N-1$. Let the complement of $\mathcal{R}^{\text {tr }}$ be the index set $\mathcal{R}$ in $\mathcal{N}=\{0,1, \ldots, N-1\}$. Therefore, $C_{k} \equiv 0 \forall k \in \mathcal{R}^{t r}$ and $C_{k}^{t r} \equiv 0 \forall k \in \mathcal{R}$. This can be expressed as:

$$
C_{k}+C_{k}^{t r}= \begin{cases}C_{k}, & k \in \mathcal{R} \\ C_{k}^{t r}, & k \in \mathcal{R}^{t r}\end{cases}
$$

The PAPR is redefined as:

$$
P A P R^{t r}=\frac{\max \left|D_{n}+D_{n}^{T R}\right|^{2}}{E\left\{\left|D_{n}\right|^{2}\right\}}
$$

The objective in tone reservation technique is to obtain $\left\{C_{k}^{t r}, k=0,1, \ldots, N-1\right\}$ such that:

$$
C^{\operatorname{tr}(\text { opt })}=\underset{\left(C^{t r} \in a_{m k}\right)}{\min } \max \left\{\left|D_{n}^{T R}\right|^{2}, n=0,1, \ldots, N-1\right\}
$$

\section{Proposed Phase Sequence Scheme}

The PN sequence is generated based on concatenating matrices. Denoting the seed matrix by $\boldsymbol{A}_{0}$ that can be expressed as:

$$
\boldsymbol{A}_{0}=\left[\begin{array}{ll}
\mathrm{a}_{1} & \mathrm{a}_{2} \\
\mathrm{a}_{3} & \mathrm{a}_{4}
\end{array}\right]
$$

where $a_{i} \in\{1,-1,+j,-j\}, i=1,2,3,4$ and $\boldsymbol{A}_{0}$ must include all the elements in the given set. Thus, one possible realization of $\boldsymbol{A}_{0}$ is: 


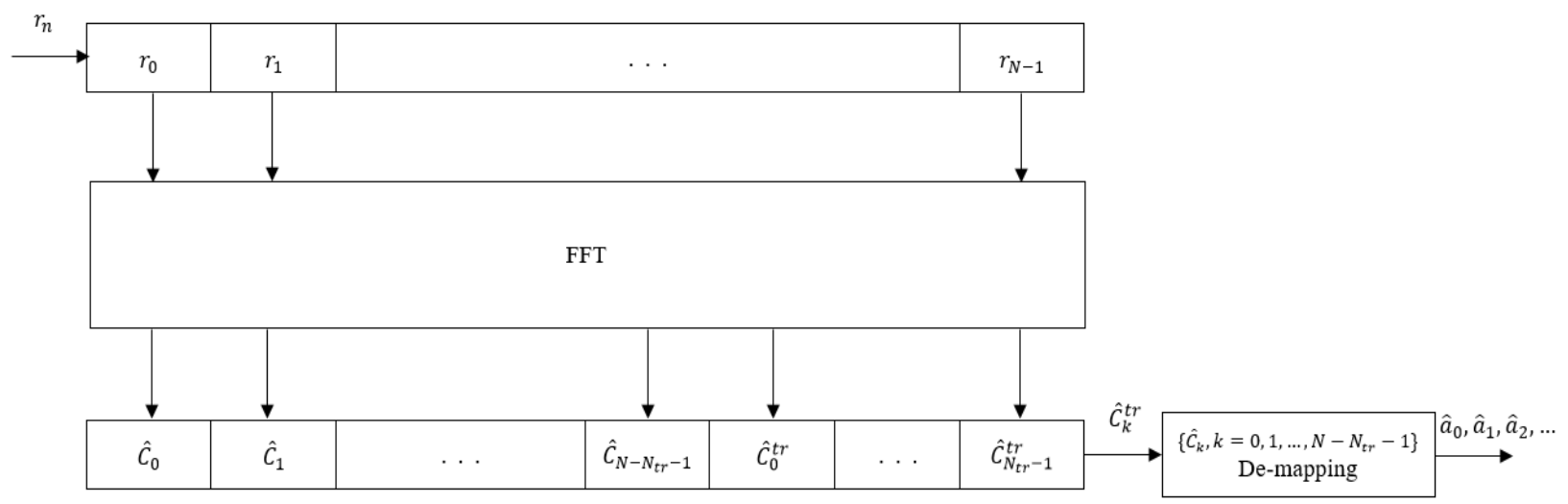

Figure 2: Block diagram for demodulation of OFDM signal with TR technique.

$$
\boldsymbol{A}_{0}=\left[\begin{array}{cc}
1 & j \\
-1 & -j
\end{array}\right]
$$

It was reported in [19] that not all available patterns of $\boldsymbol{A}_{0}$ will perform the same. Figure 3 demonstrates a sample of the possible patterns of the seed matrix $\boldsymbol{A}_{0}$.

\begin{tabular}{|c|c|c|}
\hline $\mathrm{a}$ & $\mathrm{b}$ & $\mathrm{c}$ \\
\hline$\left[\begin{array}{cc}1 & -j \\
-1 & j\end{array}\right]$ & {$\left[\begin{array}{cc}j & 1 \\
-1 & -j\end{array}\right]$} & {$\left[\begin{array}{cc}1 & -j \\
j & -1\end{array}\right]$} \\
\hline
\end{tabular}

Figure 3: Sample of the available patterns of $\boldsymbol{A}_{0}$.

In Figure 3, pattern (c) must be avoided for $\boldsymbol{A}_{0}$ while Pattern (b) performs poorer than pattern (a).

It is noted that phase sequences in the proposed TR technique can be generated using seed matrix $\boldsymbol{A}_{0}$, which is controlled by:

$$
\boldsymbol{A}_{m}=\left[\begin{array}{ll}
\boldsymbol{A}_{m-1} & \boldsymbol{A}_{m-1} \\
\boldsymbol{A}_{m-1} & \boldsymbol{A}_{m-1}^{T}
\end{array}\right], \quad m=\log _{2} N-1
$$

where, $\boldsymbol{A}_{m-1}^{T}$ is conjugate transpose of $\boldsymbol{A}_{m-1}$ and $N=2^{n}, n=$ $2,3,4, \ldots$

\section{Receiver for OFDM Signal with the TR Technique}

Figure 2 shows block diagram of receiver for detection of OFDM signal with TR method. The received signal, $r_{n}$, can be expressed as:

$$
r_{n}=D_{n}^{T R}+w_{n}, \quad n=0,1, \ldots, N-1
$$

where $w_{n}$ is AWGN with zero mean and a variance of $N_{0} / 2$.

Thus, received complex symbols, $\hat{C}_{k}^{t r}$, are given by:

$$
\hat{C}_{k}^{t r}=F F T\left\{r_{n}\right\} \quad n, k=0,1, \ldots, N-1
$$

If the AWGN noise term, $w_{n}$, in (11) is ignored, then (12) can be expressed as:

$$
\hat{C}_{k}^{t r}=\sum_{n=0}^{N-1} D_{n}^{T R} e^{-j 2 \pi k n / N}, \quad k=0,1, \ldots, N-1
$$

The useful data symbols are denoted by $\hat{C}_{k}$, which are to be extracted from $\hat{C}_{k}^{t r}$ and this can be expressed as:

$$
\hat{C}_{k}=\hat{C}_{k}^{t r}, \quad k \in \mathcal{R}
$$

\section{BER in Frequency Non-Selective Rayleigh Fading Channel}

Figure 4 shows OFDM signal received over frequency nonselective Rayleigh fading channel.

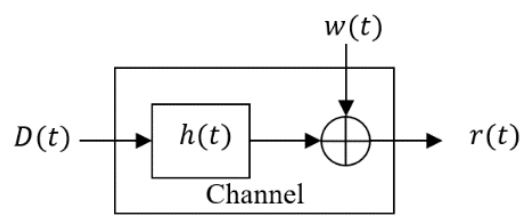

Figure 4: Model of frequency non-selective Rayleigh fading channel.

$D(t)$ in Figure 4 denotes the continuous OFDM signal of the OFDM discrete signal $D_{n}^{T R}$.

Hence, $D(t)$ is sent over the channel and therefore $r(t)$ can thus be represented as:

$$
r(t)=h(t) * D(t)+w(t) \quad 0 \leq t \leq T_{S}
$$

where $h(t)=\alpha(t) e^{j \varphi(t)}, 0 \leq t \leq T_{s}$ is the channel impulse response, $*$ denotes convolution, and $w(t)$ denotes AWGN.

For frequency non-selective Rayleigh fading channel, $h(t)$ can be modeled as a multiplicative distortion scaler to the transmitted signal $D(t)$. It is assumed that the channel is time-invariant and the impulse response of the channel is reasonably constant, at least during each symbol interval $T_{S}$ of the transmitted signal $D(t)$. The condition of the frequency non-selective is expressed as:

$$
B_{s} \ll B_{c} \text { and } T_{s} \ll \sigma_{\tau}
$$

where $B_{S}$ denotes the bandwidth and $T_{S}$ denotes the symbol duration of the transmitted signal, whereas, $B_{c}$ denotes the coherence bandwidth and the RMS delay spread is denoted by $\sigma_{\tau}$.

Thus, the channel can be expressed as:

$$
h(t)=\alpha \delta(\mathrm{t}), \quad 0 \leq t \leq T_{s}
$$


where it is assumed that $\alpha$ is estimated perfectly. Using (17), in (15)

$$
r(t)=\alpha D(t)+w(t)
$$

where $\alpha$ is the fading parameter. If $\alpha$ is fixed then the probability of error rate $P_{b}$ expression can thus be expressed as a function of the signal-to-noise ratio $\left(\gamma_{b}\right)$. For OFDM-QPSK system, the $P_{b}$ is given by [21]:

$$
P_{b}\left(\gamma_{b}\right)=Q\left(\sqrt{2 \gamma_{b}}\right)
$$

where $\gamma_{b}=\alpha^{2} \varepsilon_{b} / N_{0}$ and $Q(x)$ is the $Q$ function [21].

The average error probability of OFDM-QPSK system can be shown to be given by:

$$
P_{b_{\_} \text {Rayleigh }}=\frac{1}{2}\left(1-\sqrt{\frac{\bar{\gamma}_{b}}{\bar{\gamma}_{b}+1}}\right)
$$

\section{Numerical Results}

QPSK-OFDM systems PAPR performance were observed when the proposed TR scheme is used. One hundred thousand blocks of OFDM symbols are considered for simulation when $N=$ 128. Both the introduced PN sequence and the phase sequence presented in [10] are examined. The reserved tones' locations are chosen randomly.

Figures 5 (a-d) show the CCDFs plots of QPSK-OFDM systems PAPR performance. The PAPR performance provided are for number of subcarriers $N=128$ and when reserved tones are $N_{t r}=4,8,16$, and 32. Also, these results are tabulated in Table 1 .

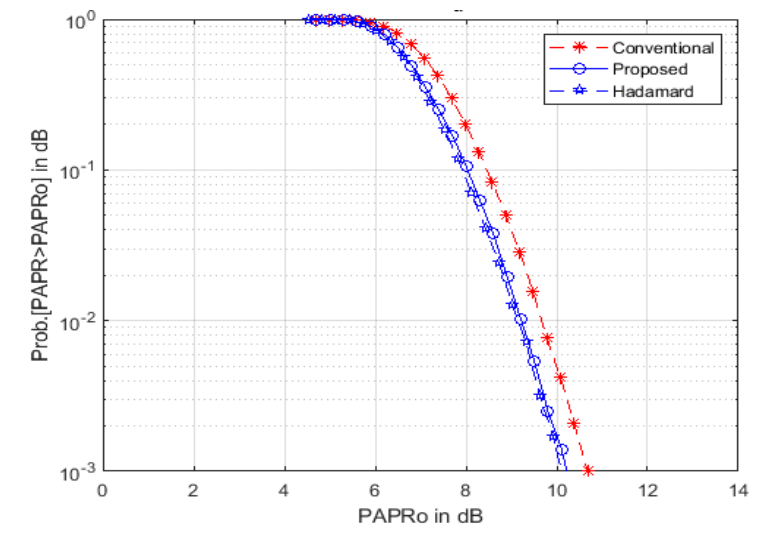

Figure 5 (a): PAPR performance of 128-subcarrier QPSK-OFDM system with 4 reserved tones

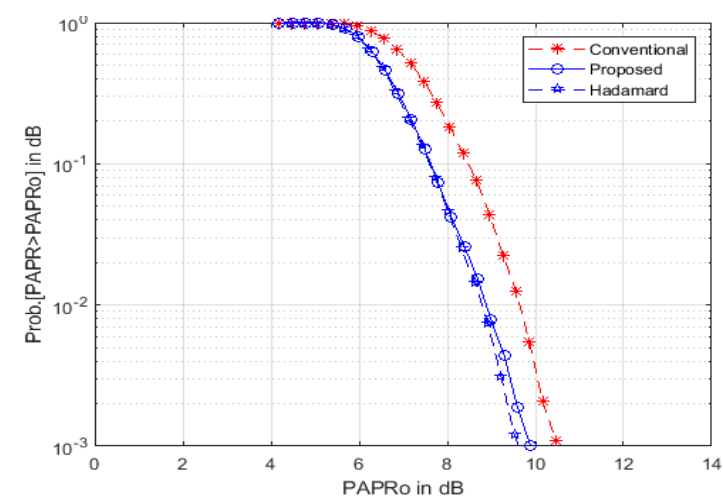

Figure 5 (b): PAPR performance of 128-subcarrier QPSK-OFDM system with 8 reserved tones
From Figures 5 (a-d) and Table 1, it is observed that the attainable PAPR performances for the two examined sequences are relatively equal. Also, it is noted that the reduction in PAPR is directly proportional to the number of reserved tones.

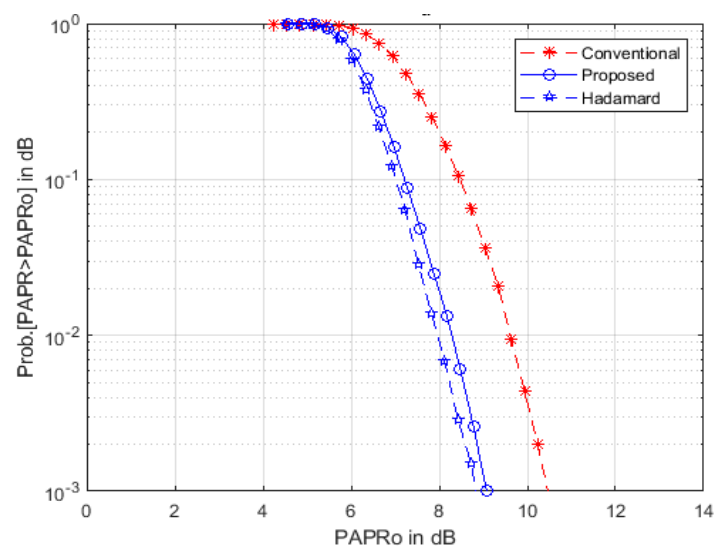

Figure 5 (c): PAPR performance of 128-subcarrier QPSK-OFDM system with 16 reserved tones

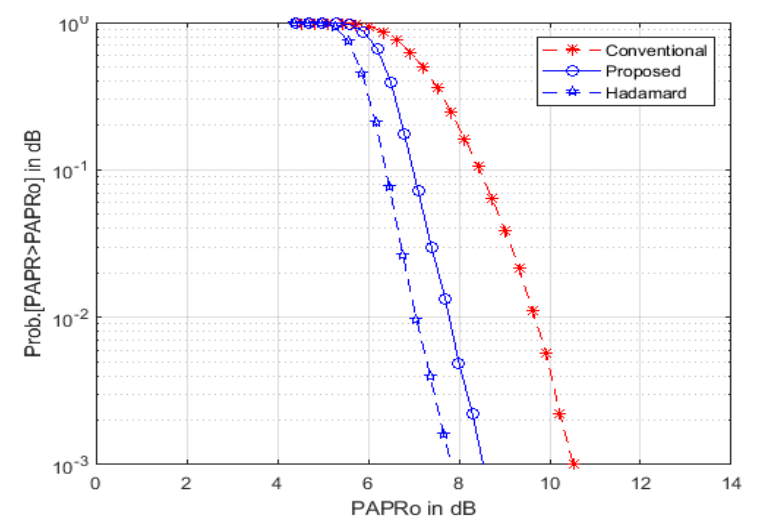

Figure 5 (d): PAPR performance of 128-subcarrier QPSK-OFDM system with 32 reserved tones

Table 1: Attainable PAPR for QPSK-OFDM system with the TR method using the proposed PN sequence and Hadamard phase sequence.

\begin{tabular}{|c|c|c|c|}
\hline$N_{t r}$ & $\begin{array}{c}\text { Attainable } \\
\text { PAPR } \\
\text { Hadamard }\end{array}$ & $\begin{array}{c}\text { Attainable } \\
\text { PAPR } \\
\text { Proposed }\end{array}$ & $\begin{array}{c}\text { Attainable } \\
\text { PAPR } \\
\text { Conventional }\end{array}$ \\
\hline 4 & $10.286 d B$ & $10.11 d B$ & \\
\cline { 1 - 3 } & $9.866 d B$ & $10.082 d B$ & \multirow{2}{*}{$10.659 d B$} \\
\hline 16 & $8.992 d B$ & $9.241 d B$ & \\
\hline 32 & $7.756 d B$ & $8.457 d B$ & \\
\hline
\end{tabular}

Figures 6 and 7 illustrate the simulated BER versus SNR curves for 128-subcarrier QPSK-OFDM system with the TR technique when using the proposed PN sequence in AWGN channel and over a frequency non-selective Rayleigh fading channel respectively. Also, these results are tabulated in Table 2 and Table 3 accordingly.

Table 2: $E_{b} / N_{0}$ required to achieve $\mathrm{BER}=10^{-3}$ for an OFDM system over the AWGN channel.

\begin{tabular}{|c|c|c|c|}
\hline Sequence & Conventional & Hadamard & Proposed \\
\hline BER & $6.758 d B$ & $6.758 d B$ & $6.769 d B$ \\
\hline
\end{tabular}




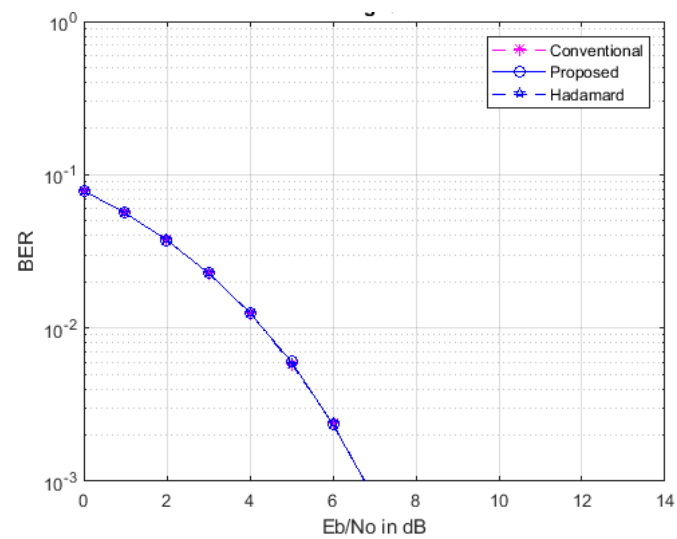

Figure. 6: BER performance of 128-subcarrier QPSK-OFDM system with proposed TR technique in AWGN channel.

Figure 7 illustrates the simulated BER versus SNR curves for 128-subcarrier QPSK-OFDM system with the proposed TR technique when using the proposed PN sequence over the frequency non-selective Rayleigh channel. Ideal channel state information (CSI) is considered.

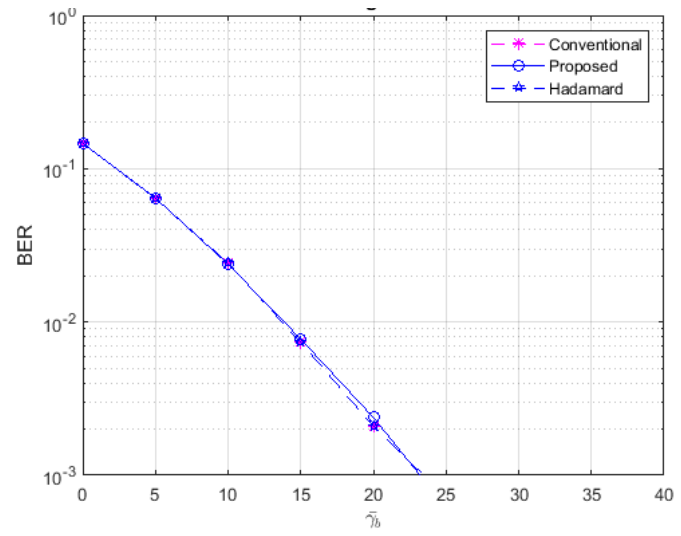

Figure 7: BER performance of 128-subcarrier QPSK-OFDM system with proposed TR technique over frequency non-selective Rayleigh fading channel.

Table 3: Average $E_{b} / N_{0}$ required to achieve $\mathrm{BER}=10^{-3} \mathrm{OFDM}$ system over the frequency non-selective channel.

\begin{tabular}{|c|c|c|c|}
\hline Sequence & Conventional & Hadamard & Proposed \\
\hline BER & $22.936 d B$ & $22.936 d B$ & $23.504 d B$ \\
\hline
\end{tabular}

Figure 6 and Table 2 show that the BER performance over AWGN channel has not been degraded when the proposed PN sequence is used by the TR technique to reduce the PAPR in an OFDM system. In addition, an insignificant BER performance degradation is observed when the proposed PN sequence is used by the proposed TR method to reduce the PAPR in an OFDM system over frequency non-selective Rayleigh fading channel as shown in Figure 7 and tabulated in Table 3.

Figure 8 shows a PAPR performance comparison between QPSK-OFDM system with 16-reserved tones and QPSK-OFDM system with SLM method when the search for the optimum phase sequence is limited to 16 .

Figure 8 and Table 4 illustrate that there is a $1.288 \mathrm{~dB}$ reduction in PAPR when TR technique is used compared to 2.497 $d B$ when SLM technique is used. TR technique has only reserved 16-subcarriers for PAPR reduction however no SI is required at the receiver. In comparison to TR technique, SLM requires $\mathrm{N}$ multiplications in each iteration and it requires sending SI to the receiver.

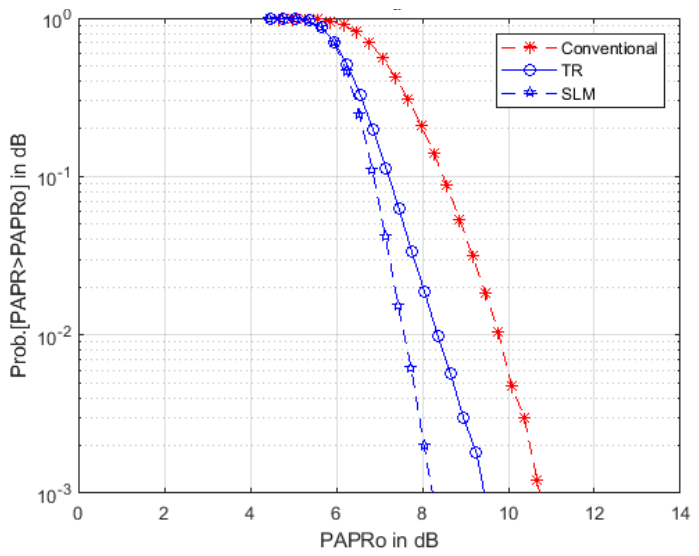

Figure 8: PAPR performance of 128-subcarrier QPSK-OFDM system with 16 reserved tones vs PAPR performance 128-subcarrier QPSK-OFDM system with SLM.

Table 4: A Comparison of the PAPR performance between 128-subcarriers QPSKOFDM system with 16-reserved tones TR technique and 128-subcarriers QPSKOFDM system with SLM technique

\begin{tabular}{|c|c|c|c|}
\hline Sequence & Conventional & TR & SLM \\
\hline PAPR & $10.725 d B$ & $9.437 d B$ & $8.228 d B$ \\
\hline
\end{tabular}

\section{Conclusion}

In this paper, a novel low-complexity TR technique for reduction of PAPR in a QPSK-OFDM system is presented and investigated. The proposed PAPR reduction scheme is easy-toimplement and has low-complexity. Also, the proposed TR technique requires no SI to be conveyed to the receiver. The simulation results demonstrate that the PAPR performance of the proposed and conventional TR methods are the same. The BER performance of the proposed system over AWGN and frequency non-selective channel have not been compromised. When PAPR performance of the proposed TR method is compared with that of SLM technique, the SLM technique outperformed the proposed TR technique by approximately $\approx 1.2 \mathrm{~dB}$. However, the complexity of SLM method is higher and requires sending SI to the receiver.

\section{Conflict of Interest}

The authors declare no conflict of interest.

\section{References}

[1] U. Reimers, "Digital video broadcasting," IEEE Communications Magazine, 36 (6), 104-110, 1998. https://doi.org/10.1109/35.685371

[2] R. van Nee, G. Awater, M. Morikura, H. Takanashi, M. Webster and K. W. Halford, "New high-rate wireless LAN standards," IEEE Communications Magazine, 37(12), 82-88, 1999. https://doi.org/ 10.1109/35.809389

[3] I. Koffman and V. Roman, "Broadband wireless access solutions based on OFDM access in IEEE 802.16," IEEE Communications Magazine, 40(4), 96-103, 2002. https://doi.org/10.1109/35.995857

[4] W. Bolton, Y. Xiao and M. Guizani, "IEEE 802.20: mobile broadband wireless access," IEEE Wireless Communications, 14(1), 84-95, 2007. https://doi.org/10.1109/MWC.2007.314554 
[5] Y. Tsai, G. Zhang, D. Grieco, F. Ozluturk and X. Wang, "Cell search in 3GPP long term evolution systems," IEEE Vehi. Tech. Magazine, 2(2), 2329, 2007. https://doi.org/10.1109/MVT.2007.912929

[6] A. Elnashar and M. A. El-Saidny, "Looking at LTE in Practice: A Performance Analysis of the LTE System Based on Field Test Results," IEEE Vehicular Technology Magazine, 8(3), 81-92, 2013. https://doi.org/10.1109/MVT.2013.2268334

[7] X. Li and L. J. Cimini, "Effects of clipping and filtering on the performance of OFDM," IEEE Communications Letters, 2(5), 131- 133, 1998. https://doi.org/10.1109/4234.673657

[8] S. Cha, M. Park, S. Lee, K. Bang and D. Hong, "A new PAPR reduction technique for OFDM systems using advanced peak windowing method," in IEEE Transactions on Consumer Electronics, 54(2), 405-410, 2008. https://doi.org/10.1109/TCE.2008.4560106

[9] S. H. Müller and J. B. Huber, "A Comparison of Peak Power Reduction Schemes for OFDM," in IEEE GLOBECOM Conference, Phoenix, AZ, 1997. https://doi.org/10.1109/GLOCOM.1997.632501

[10] R. W. Bauml, R. F. H. Fischer and J. B. Huber, "Reducing the peak-toaverage power ratio of multicarrier modulation by selected mapping," Electronics Letters, 32(22), 2056-2057, 1996. https://doi.org/ 10.1049/el: 19961384

[11] B. S. Krongold and D. L. Jones, "PAR reduction in OFDM via active constellation extension," IEEE Transactions on Broadcasting, vol. 49(3), 258-268, 2003. https://doi.org/10.1109/TBC.2003.817088

[12] S. Muller and J. Huber, "OFDM with reduced peak-to-average power ratio by optimum combination of partial transmit sequences," Electronics Letters , 33(5), 368 - 369, 1997. https://doi.org/10.1049/el:19970266

[13] L. Cimini and N. Sollenberger, " Peak-to-average power ratio reduction of an OFDM signal using partial transmit sequences," IEEE Comm. Letters , 4(3), 86 - 88, 2000. https://doi.org/10.1109/4234.831033

[14] J. Tellado, "Peak to Average Power Reduction for Multicarrier Modulation," Ph.D Thesis, Stanford University, 2000.

[15] A. Jones, T. Wilkinson and S. Barton, "Block coding scheme for reduction of peak to mean envelope power ratio of multicarrier transmission schemes," Electronics Letters, 30(25), $2098 \quad$ - 2099, 1994. https://doi.org/10.1049/el:19941423

[16] Seung Hee Han and Jae Hong Lee, "An overview of peak-to-average power ratio reduction techniques for multicarrier transmission," in IEEE Wireless $\begin{array}{llll}\text { Communications, } & 12(2), & 56-65, & \end{array}$ https://doi.org/10.1109/MWC.2005.1421929

[17] T. Jiang and Y. Wu, "An Overview: Peak-to-Average Power Ratio Reduction Techniques for OFDM Signals," in IEEE Transactions on $\begin{array}{lll}\text { Broadcasting, 54(2), 257-268, } 2008 . & .\end{array}$ https://doi.org/10.1109/TBC.2008.915770

[18] Y. Rahmatallah and S. Mohan, "Peak-To-Average Power Ratio Reduction in OFDM Systems: A Survey And Taxonomy," in IEEE Communications Surveys \& Tutorials, 15(4), 1567-1592, 2013. https://doi.org/10.1109/SURV.2013.021313.00164

[19] M. Ali, R. K. Rao and V. Parsa, "PAPR Reduction in OFDM System Using New Method for Generating Pseudo-Random Sequence for SLM Technique" in $31^{\text {st }}$ Annual IEEE Canadian Conference on Electrical \& Computer Engineering (CCECE), Quebec City, Canada, 2018. https://doi.org/10.1109/CCECE.2018.8447835

[20] L. Wang and C. Tellambura, "Analysis of Clipping Noise and ToneReservation Algorithms for Peak Reduction in OFDM Systems," in IEEE Transactions on Vehicular Technology, 57(3), 1675-1694, 2008. https://doi.org/10.1109/TVT.2007.907282

[21] J. Proakis, digital communications, McGraw-Hill, 1995 